DOI: $10.4274 /$ jarem.galenos.2021.77528

J Acad Res Med 2021;11(3):283-7

\title{
Safety Evaluation of Early Active and Passive Motion Without Immobilization in Metacarpal Fractures
}

\author{
(1) Betül Başar1, (1) Abdulkadir Polat2 \\ ${ }^{1}$ University of Health Sciences Turkey, Gaziosmanpaşa Training and Research Hospital, Clinic of Physical Medicine and Rehabilitation, \\ İstanbul, Turkey \\ 2University of Health Sciences Turkey, Gaziosmanpaşa Training and Research Hospital, Clinic of Orthopaedics and Traumatology, İstanbul, \\ Turkey
}

Cite this article as: Başar B, Polat A. Safety Evaluation of Early Active and Passive Motion Without Immobilization in Metacarpal Fractures. J Acad Res Med 2021;11(3):283-7

\begin{abstract}
Objective: Early motion after surgical treatment of metacarpal fractures is important to prevent joint motion limitation. Contrarily, the loss of reduction in the fracture line with an early motion negatively affects the results. This study aimed to evaluate the reliability of early active and passive motion without immobilization after surgical treatment and compare the results with the traditional rehabilitation program.

Methods: This study included 86 patients who were fixed with miniplate screws. Patients were divided into two groups according to the rehabilitation programs. The first group had immobilization with orthosis and traditional rehabilitation program for 4 weeks, whereas the second group had an early active and passive joint range of motion exercises without immobilization. The first group included 37 males and 16 females with a mean age of $34.92 \pm 12.97$ years who were followed for an average of $43.19 \pm 13.03$ months. The second group included 23 males and 16 females with a mean age of $31.82 \pm 11.92$ years who were followed for an average of $39.05 \pm 12.74$ months.

Results: No significant differences were determined between the groups in terms of age, female-male ratio, time from injury to surgery, follow-up period, fractured extremity, and dominant-non-dominant ratio. Additionally, no significant differences were found between the groups in terms of grip strength and time to return to work at the final follow-up. The total joint range of motion and Quick Disabilities of the Arm, Shoulder, and Hand scores were significantly better in the second than the first group. Union at the fracture line without reduction loss was observed in both groups at the final follow-up.

Conclusion: Fixation with miniplate screws provides stable fixation in extra-articular metacarpal fracture. Early active and passive motion without immobilization does not cause complications at the fracture line with this stable fixation. Contrarily, more successful results were obtained than the traditional rehabilitation program in terms of the range of motion and functional results.
\end{abstract}

Keywords: Metacarpal fracture, rehabilitation, early active motion, early passive motion

ORCID IDs of the authors: B.B. 0000-0003-3547-2537; A.P. 0000-0002-3224-7134.

Corresponding Author: Betül Başar,

E-mail: betulakarvardar@hotmail.com
Received Date: 13.10.2021 Accepted Date: 30.11.2021

(C) Copyright 2021 by University of Health Sciences Turkey, Gaziosmanpaşa Training and Research Hospital. Available on-line at www.jarem.org 


\section{INTRODUCTION}

Hand fractures are the most common fractures in the human skeleton. Metacarpal fractures constitute $30-50 \%$ of hand fractures (1), which are more common in young adults (2). Patients are frequently encountered in the rehabilitation clinics with the expectation of early return to work after the fracture (3). Consensus is unavailable on the optimal treatment option for extra-articular metacarpal fractures. However, prolonged immobilization carries a potential risk of joint stiffness in closed reduction casting and pinning $(4,5)$. Fixation with miniplate screws provides a rigid and stable fixation for early motion (4). However, a risk of motion limitation may develop due to friction and adhesions caused by plates and screws (6). Tendon adhesions, joint motion limitation, and strength and functional loss can develop after open surgery. Early postoperative rehabilitation is effective in preventing these complications (7). Early rehabilitation aimed to achieve a more successful result in joint range of motion and functional result, to provide an early return to normal life and work-life, and to prevent tendon adhesions (8). However, the loss of reduction in the fracture line due to early forced action negatively affects the results (9). Therefore, early passive motion is not started in the traditional rehabilitation program, but an only early active movement. The patient also uses a splint or orthosis for immobilization (10).

We hypothesize that early active and passive motion without immobilization will provide better results in both joint ranges of motion and functional results. This study aimed to compare early active and passive motion without immobilization with traditional rehabilitation in clinical and functional aspects.

\section{METHODS}

This study retrospectively evaluated 144 patients who are fixed with 2.0 and $2.4 \mathrm{~mm}$ miniplate screws between January 2016 and June 2020 due to extra-articular metacarpal fracture. Patients with first metacarpal fracture and those under 18 years of age as well as 19 patients who did not come for regular control, 11 who could not be regularly rehabilitated, 28 who had an additional fracture in the upper extremity before surgery or during follow-up, open fractures, and tendon, nerve, and vascular repair with fracture were excluded from our study. Final controls of 86 patients were performed.

The study protocol was approved by the University of Health Sciences Turkey Ethics Committee of Gaziosmanpaşa Training and Research Hospital (approval number: 302-2021).

Patients were evaluated under two groups according to the rehabilitation program. The first group had immobilization with the orthosis and traditional rehabilitation program for 4 weeks, whereas the second group had no immobilization and early active and passive joint range of motion was started. The coarse clothing of patients was reduced in both groups, and rehabilitation was started under the guidance of a physiotherapist on postoperative day 3. The incision area was massaged with lotion, three times for 10 min, every day after the stitches were removed. Elevation, cold application, and compression bandages were applied to reduce edema formation. The decongestive exercise was started. The hand was placed higher than the elbow and the elbow higher than the shoulder, and the affected hand was massaged starting from the fingertips to the axilla.

Orthosis was used for the whole day for 4 weeks in the first group. Additionally, the metacarpal bones were immobilized in the intrinsic plus position [wrist in 30 degrees extension, metacarpophalangeal (MCP) joints in 70-degree flexion, and proximal interphalangeal (PIP) joints in full extension]. Immobilization was performed with one finger on the radial and ulnar sides of the fractured finger. Only one adjacent finger was included in the immobilization in the $2^{\text {nd }}$ and $5^{\text {th }}$ metacarpal fractures. Contrarily, immobilization was not applied in the second group. Active joint range of motion was started in the first group under the guidance of a physiotherapist after postoperative day 3. Isometric exercises were started for muscle strengthening in the orthosis. Isometric exercises were started in the second week to strengthen the intrinsic muscles. Paraffin treatment for 20 min before the exercises was started 2 days after the sutures were removed. Active joint range of motion exercises under the guidance of a physiotherapist for 45 min was applied for 5 days in a week in the first 2 weeks and 3 days in a week in weeks 3 and 4 . Passive range of motion exercises and gentle ball squeezes and dough exercises were started for muscle strengthening at week 4. Additionally, gentle and resistance abduction exercises were started. Active joint range of motion exercises against resistance was started for muscle strengthening after week 6.

Active, actively assisted, and passive joint range of motion exercises and isometric exercises for muscle strengthening was started under the guidance of a physiotherapist in the second group after the postoperative day 3. Paraffin treatment for 20 min before the exercises was started 2 days after the sutures were removed. Active, active assisted, and passive joint range of motion exercises for 45 min was applied for 5 days a week in the first 2 weeks and 3 days a week in weeks 3 and 4 . Hand therapeutic dough and grip strengthening exercises for 10 min were started in each session in addition to the range of motion exercises after postoperative week 2. Isometric exercises were also started to strengthen the intrinsic muscles in the second week. The home exercise program was explained to the patients by an experienced physiotherapist on the contralateral intact extremity before hospital discharge. Additionally, informative brochures were given to the patients and were recommended to perform a 30-min home exercise program after 10 min of hot application twice a day.

The total joint range of motion (TJROM) and grip strength were evaluated. TJROM measurements were made by recording the sum of the flexion and extension angles of the MCP, PIP, and distal interphalangeal joints with the aid of a goniometer while the hand was in full flexion and full extension (11). Handgrip strength was measured with a Jamar dynamometer (Asimow Engineering, Los Angeles, USA) in the shoulder with $0^{\circ}$ adduction, elbow with $90^{\circ}$ flexion, and forearm at neutral rotation. Each measurement was repeated three times and the average was recorded as $\mathrm{kg}$ (8). Quick Disabilities of the Arm, Shoulder, and Hand (Q-DASH) scoring was used for satisfaction assessment (3). The time to return 
to work was evaluated through the Social Security Institution's incapacity report system.

\section{Statistical Analysis}

Statistical analyses were performed by Statistical Package for the Social Sciences version 20 computer software. All measured data are descriptively presented. Data were presented as numbers, mean, and standard deviation. The Shapiro-Wilk test was used to determine normal data distribution. Differences in clinical details were assessed using the chi-squared test for categorical variables, such as gender, hand side, hand dominance, and affected finger, and the Student's t-test for continuous variables, such as age, period injury to operation, and return to work period. The relationship between the rehabilitation modalities and TJROM, grip strength, Q-DASH, and return to work period were analyzed using the tests (Samples t, Mann-Whitney U, chi-square, Fisher's Exact). The significance level was considered at $p$-values of $<0.05$.

\section{RESULTS}

No statistically significant differences were found between the groups in terms of age, gender, time from injury to surgery, fractured extremity side, dominant-nondominant extremity ratio, and mean follow-up time ( $p>0.05$; Table 1).

Handgrip strength was $40.87 \pm 6.41 \mathrm{~kg}$ in the first group and $39.20 \pm 11.52 \mathrm{~kg}$ in the second group at the final follow-up, without statistically significant differences between the groups $(p=0.4066$; Table 2). The mean TJROM score was $229.80 \pm 15.99^{\circ}$ in the first group and $244.05 \pm 12.88^{\circ}$ in the second group. The mean Q-DASH score was $3.15 \pm 3.53$ in the first group and $0.98 \pm 1.91$ in the second group. The results of TJROM and Q-DASH were statistically significantly better in the second group than in the first group $(p<0.05)$. The mean time to return to work was $32.19 \pm 8.94$ days in the first group, whereas $29.58 \pm 6.63$ days in the second group, without statistically significant difference $(p=0.1259)$. Proximal humerus fracture was observed in one patient due to falling in 22 days in the second group. The reoperated patient was excluded from the study. One patient had delayed wound healing in the first group. The dressing was followed up with rehabilitation after the stitches were removed. The wound completely healed without the need for extra intervention within 30 days.

\section{DISCUSSION}

The hand is a region where many movements are intertwined in a small area. Minor injuries can result in a major functional loss (12). A wide variety of treatment options are available for metacarpal fractures $(13,14)$. Surgical treatment is preferred in fractures with high rotational displacement and shortness at the fracture line. Surgery is also applied in multipart fractures and displaced fractures, which cannot be closely reduced.

Miniplate screw system has developed a lot with implant technology developments. Starting early motion after fixation with miniplate screws in metacarpal fractures was possible. Gaining an early joint range of motion shortens the patient's return to work days $(11,15)$. Early motion after rigid fixation is ideal in metacarpal fractures; however, it is not without problems (16).

\section{Table 1. Demographic values, period from injury to surgery, and mean follow-up period of the patients}

\begin{tabular}{|l|l|l|l|}
\hline & First group & Second group & p-values \\
\hline Age (years) & $34.91 \pm 12.97$ & $31.82 \pm 11.92$ & 0.2531 \\
\hline Male/female & $31 / 16$ & $23 / 16$ & 0.6578 \\
\hline Right hand/left hand & $30 / 17$ & $29 / 10$ & 0.3097 \\
\hline Dominant/non-dominant & $32 / 15$ & $31 / 8$ & 0.3449 \\
\hline Effected finger & & & \\
\hline $2^{\text {nd }}$ metacarpal & 6 & 6 & 0.8271 \\
\hline $3^{\text {rd }}$ metacarpal & 11 & 6 & 0.3653 \\
\hline $4^{\text {th }}$ metacarpal & 9 & 8 & 0.1416 \\
\hline $5^{\text {th }}$ metacarpal & 21 & 19 & $2.75 \pm 2.05$ \\
\hline Period injury to operation & $2.36 \pm 1.88$ & $39.05 \pm 12.74$ & \\
\hline Mean follow-up period & $43.19 \pm 13.03$ & & \\
\hline
\end{tabular}

Table 2. Grip strength, range of motion, functional results, and time taken to return to work at final follow-ups

\begin{tabular}{|l|l|l|l|}
\hline & First group & Second group & p-values \\
\hline Grip strength $(\mathbf{k g})$ & $40.87 \pm 6.41$ & $39.20 \pm 11.52$ & 0.4066 \\
\hline Total joint range of motion & $229.80 \pm 15.99$ & $244.05 \pm 12.88$ & $<0.0001$ \\
\hline Quick disabilities of the arm, shoulder, and hand & $3.15 \pm 3.53$ & $0.98 \pm 1.91$ & 0.0005 \\
\hline Return to work period & $32.19 \pm 8.94$ & $29.58 \pm 6.63$ & 0.1259 \\
\hline
\end{tabular}


Adhesions and plate screw irritations may occur in the extensor tendons after fixation (17). Complication rates after metacarpal fractures are between $32 \%$ and $36 \%$. Joint stiffness is the most frequently reported complication with a rate of $76 \%$. TJROM has been reported to be $<220^{\circ}$ in these patients (18).

The literature demonstrated that early motion can be started after rigid fixation with miniplate screws (4). Immobilization is applied in the first 2 weeks to prevent potential reduction loss in the traditional rehabilitation program (3). Active motion exercises are under the direct control of the patient through osseous structures and musculotendinous structures. Active motion exercises generate tendon gliding, promote strength, endurance, and exchange lymphatic drainage. Passive motion exercises are applied by the physiotherapist to overcome tissue resistance and are not under the control of the patient, which is usually painful and is not applied early in classical rehabilitation to avoid tissue injuries and possible implant failure.

An animal study revealed that early controlled passive movement in unstable extra-articular metacarpal fractures helps to create a more stable biomechanically union without disturbing the callus tissue and reduces dorsal angulation at the fracture line. Additionally, an early controlled passive motion was determined to cause a mechanical stimulus that triggered healing and tissue differentiation at the fracture line (19). A biomechanical study has determined that the use of miniplate screws in metacarpal fractures is sufficiently resistant to controlled passive motion exercise cyclic loads (20).

Implant failure was not observed in patients who underwent traditional rehabilitation and in patients who started active, actively assisted, and passive joint range of motion exercises after the postoperative day 3 in our study. An early passive motion was determined to be started after osteosynthesis with miniplate screws.

Success after hand rehabilitation is associated with pain, strength gain, and total active range of motion, and functional gain. The mean handgrip strength at week 6 was 33.9 (22-51) kg in patients who underwent immobilization for 1 week after surgical treatment (21). The clinical results of the classical rehabilitation program and home exercise program were compared in patients who underwent fixation with plate screws in a study. An average of $35 \mathrm{~kg}$ grip strength was reached in both groups at the end of 12 weeks (3). The mean grip strength was $39.20 \pm 11.52 \mathrm{~kg}$ in patients who were not immobilized but applied early passive and active exercise, whereas $40.87 \pm 6.41 \mathrm{~kg}$ in patients who underwent conventional rehabilitation in our study. No significant differences were found in the handgrip strength between the two groups. The mean handgrip values of our study, which were higher than the values of both studies, are thought to be associated with a longer follow-up period.

The mean TJROM value was evaluated according to the rehabilitation applied in a study conducted with patients who underwent fixation with miniplate screws, which was $256^{\circ}$ in patients who received the home program and $245^{\circ}$ in patients who underwent rehabilitation under the guidance of a physiotherapist. Significantly, better results were achieved in patients who underwent rehabilitation under the guidance of a physiotherapist (3). Another study evaluated 54 metacarpal fractures of 42 patients who were immobilized with an orthosis in the intrinsic plus position that allows finger movement and revealed a mean TJROM of $241^{\circ}$ at week 6 and $253^{\circ}$ at week 12 (5). A study evaluated closed reduction percutaneous pinning in 33 patients who were immobilized for $30.9 \pm 5.8$ days and revealed a mean TJROM of $249 \pm 40^{\circ}$ at $2.9 \pm 2.4$ months. Additionally, open reduction and plate screws fixation was performed in 23 patients who were immobilized for $20 \pm 5.6$ days and revealed a mean TJROM of $234.3 \pm 58.5^{\circ}$ at $4.2 \pm 5.6$ months (4). The mean TJROM was $244.05 \pm 12.88^{\circ}$ in patients who underwent early passive and active exercise without immobilization in our study. The mean TJROM was $229.80 \pm 15.99^{\circ}$ in our patients who underwent conventional rehabilitation. TJROM value was significantly better in patients who applied early passive and active exercise without immobilization.

A study clinically evaluated 37 metacarpal fractures fixed with plate screws, wherein the patients underwent a traditional rehabilitation program with immobilization after surgical treatment. The mean Q-DASH score was 3.6 at 32 months (22). In our study, the mean Q-DASH score was $3.15 \pm 3.53$ in patients who underwent conventional rehabilitation. Similar results were obtained in Q-DASH scores with this study. Better Q-DASH results $(0.98 \pm 1.91)$ were obtained in patients who started early active and passive motion without immobilization. Starting early active and passive motion without immobilization was revealed to give better results than traditional rehabilitation in our study.

\section{Study Limitations}

The rehabilitation of patients was started in the same rehabilitation center; however, the rehabilitation of patients after hospital discharge continued under the guidance of different physiotherapists. Different surgeons performed the operations. The metacarpal fracture fixation was performed with miniplate screws; however, the same brand of the implant was not used in all patients. The randomization method was not used in the distribution of patients into groups. Pretreatment values are unknown because prefracture evaluations of patients could not be made. No difference was found between the groups in terms of age, male-female ratio, and dominant extremity involvement, thus no significant difference was found between the groups in terms of pretreatment values.

\section{CONCLUSION}

Fixation with miniplate screws provides a stable fixation in extra-articular metacarpal fractures. This stable fixation allows passive, as well as active motion, in the early period without immobilization. Initiation of active and passive motion in the early period without immobilization provides better functional results and joint range of motion. Our study determined that early active 
8. Hays PL, Rozental TD. Rehabilitative strategies following hand fractures. Hand Clin 2013; 29: 585-600

and passive motion without immobilization is required to achieve more successful results after fixation of extra-articular metacarpal fractures with miniplate screws.

Ethics Committee Approval: The study protocol was approved by the Ethics Committee of Gaziosmanpaşa Training and Research Hospital (decision no: 302, date: 23.06.2021)

Informed Consent: Retrospective study.

Peer-review: Externally peer-reviewed.

\section{Authorship Contributions}

Surgical and Medical Practices - B.B., A.P.; Concept - B.B.; Design - B.B.; Data Collection or Processing - B.B., A.P.; Analysis or Interpretation - B.B.; Literature Search - A.P.; Writing - B.B., A.P.

Conflict of Interest: No conflict of interest was declared by the authors.

Financial Disclosure: The authors declared that this study received no financial support.

\section{REFERENCES}

1. McNemar TB, Howell JW, Chang E. Management of metacarpal fractures. J Hand Ther 2003; 16: 143-51.

2. Ben-Amotz O, Sammer DM. Practical management of metacarpal fractures. Plast Reconstr Surg 2015; 136: 370e-379e. doi: 10.1097/ PRS.0000000000001527.

3. Gülke J, Leopold B, Grözinger D, Drews B, Paschke S, Wachter NJ. Postoperative treatment of metacarpal fractures-Classical physical therapy compared with a home exercise program. J Hand Ther 2018; 31: $20-28$.

4. Vasilakis V, Sinnott CJ, Hamade M, Hamade H, Pinsky BA. Extra-articular metacarpal fractures: closed reduction and percutaneous pinning versus open reduction and internal fixation. Plast Reconstr Surg Glob Open 2019;7:e2261. doi: 10.1097/GOX.00000000000002261.

5. Al-Qattan MM. Outcome of conservative management of spiral/long oblique fractures of the metacarpal shaft of the fingers using a palmar wrist splint and immediate mobilisation of the fingers. J Hand Surg Eur Vol 2008; 33: 723-7.

6. García-Medrano B, Honecker S, Facca S, Simón FP, Delgado PJ, Liverneaux P. Intramedullary percutaneous fixation of metacarpal fractures: screw versus connected K-wires. Eur J Orthop Surg Traumatol 2021 May 17. doi: 10.1007/s00590-021-02977-3. Epub ahead of print.

7. Patil A, Zeeshan Vasif M, Gaya BT. Complications in surgical management of phalangeal and metacarpal fractures. Int J Orthop Sci 2019; 5: 681-3.
9. Duncan RW, Freeland AE, Jabaley ME, Meydrech EF. Open hand fractures: Surg Am 1993; 18: 387-94.

10. Jun D, Bae J, Shin D, Choi H, Kim J, Lee M. Controlled active exercise after open reduction and internal fixation of hand fractures. Arch Plast Surg 2021; 48: 98-106.

11. Başar H, Başar B, Başçı O, Topkar OM, Erol B, Tetik C. Comparison of treatment of oblique and spiral metacarpal and phalangeal fractures with mini plate plus screw or screw only. Arch Orthop Trauma Surg 2015; 135 : 499-504.

12. Kamath JB, Harshvardhan, Naik DM, Bansal A. Current concepts in managing fractures of metacarpal and phalangess. Indian J Plast Surg 2011; 44: 203-11.

13. Deutsch C, Maklad M, Delikonstantinou I, Sood M. The management of metacarpal fractures: a retrospective review of outcomes from a highvolume centre. J Orthop Surg 2020; 4: 27-31.

14. Hooper RC, Chen JS, Kuo CF, Chung KC. Closed metacarpal neck fractures: a review of resource use in operative and nonoperative management. Plast Reconstr Surg 2020; 146: 572-9.

15. Carender CN, Buckwalter JA, Glass NA, Westermann RW. Descriptive epidemiology and return to sport after hand fractures in NCAA athletes. lowa Orthop J 2019; 39: 49-54

16. Baumgartner RE, Federer AE, Guerrero EM, Mithani SK, Ruch DS, Richard MJ. Complications of low-profile plate fixation in metacarpal fractures. Orthopedics 2021; 44: e91-4. doi: 10.3928/01477447-20200925-02.

17. Dyrna FGE, Avery DM 3rd, Yoshida R, Lam D, Oeckenpöhler S, Cote MP, Obopilwe E, Rodner CM, Mazzocca AD. Metacarpal shaft fixation: compression screws. BMC Musculoskelet Disord 2021; 22: 335.

18. Kollitz KM, Hammert WC, Vedder NB, Huang JI. Metacarpal fractures: treatment and complications. Hand (N Y) 2014; 9: 16-23.

19. Feehan LM, Tang CS, Oxland TR. Early controlled passive motion improves early fracture alignment and structural properties in a closed extra-articular metacarpal fracture in a rabbit model. J Hand Surg Am 2007; 32: 200-8.

20. Doht S, Meffert RH, Raschke MJ, Blunk T, Ochman S. Biomechanical analysis of the efficacy of locking plates during cyclic loading in metacarpal fractures. ScientificWorldJournal 2014; 2014: 648787.

21. Moon SH, Kim HS, Jung SN, Kwon H. The efficacy of transverse fixation and early exercise in the treatment of fourth metacarpal bone fractures. Arch Plast Surg 2016; 43: 189-96.

22. Aykut S, Öztürk K, Özcan Ç, Demiroğlu M, Gürün AU, Özden E. Results of surgical treatment in metacarpal shaft fractures using low profile mini plates. Ulus Travma Acil Cerrahi Derg 2015; 21: 279-84. an analysis of the recovery of active motion and of complications. $J$ Hand a biomechanical comparison of dorsal plating, lag screws, and headless 